\title{
Safe use of hepatitis B surface antigen positive grafts in liver transplantation: a nationwide study based on Korean Organ Transplantation Registry Data
}

\author{
YoungRok Choi ${ }^{1}$, Kwang-Woong Lee ${ }^{1}$, Bong-Wan Kim² ${ }^{2}$ Dong-Sik Kim³ ${ }^{3}$, Jong Man Kim ${ }^{4}$ Yang Won $\mathrm{Nah}^{5}$, Shin Hwang ${ }^{5}$, \\ Jae Geun Lee ${ }^{6}$, Je Ho Ryu ${ }^{7}$, Geun Hong ${ }^{8}$
}

\footnotetext{
${ }^{1}$ Department of Surgery, Seoul National University College of Medicine, Seoul, Korea

${ }^{2}$ Department of Surgery, Ajou University Hospital, Suwon, Korea

${ }^{3}$ Department of Surgery, Korea University College of Medicine, Seoul, Korea

${ }^{4}$ Department of Surgery, Sungkyunkwan University School of Medicine, Seoul, Korea

${ }^{5}$ Department of Surgery, University of Ulsan College of Medicine, Seoul, Korea

${ }^{6}$ Department of Surgery, Yonsei University College of Medicine, Seoul, Korea

${ }^{7}$ Department of Surgery, Pusan National University School of Medicine, Yangsan, Korea

${ }^{8}$ Department of Surgery, Ewha Womans University School of Medicine, Seoul, Korea
}

Background: Liver grafts from donors with chronic hepatitis B virus (HBV) infection has expanded the donor pool in HBV endemic area. Endeavor to use HBsAg (+) graft as safe alternatives were kept up for several years, and the outcome has improved by using hepatitis $B$ virus immune globulin (HBIG) and nucleoside analogs (NA).

Methods: Among 4,265 liver transplantations (LTs) registered in the KOTRY database prospectively between April 2014 and January 2020,39 cases $(0.9 \%)$ of LTs using HBsAg $(+)$ grafts were identified. We compared outcomes and its associating factors in LT using HBsAg (+) grafts compared to HBsAg $(-)$ grafts $(n=3,971)$.

Results: Twenty deceased donor LT (DDLT-HBV) and 19 living donor LT (LDLT-HBV) were performed using HBsAg (+) grafts. They maintained HBIG or NA, or both in the perioperative period. The mean end-stage liver disease (MELD) score was 15.8 \pm 9.2 , and the follow-up period was $28.0 \pm 19.2$ months. Six LTs were performed for patients in intensive care unit. There was no difference in the patients' survival between the two groups $(\mathrm{P}=0.111)$. In addition, no difference between LDLT-HBV and DDLT-HBV was observed $(P=0.885)$. Fifteen patients (38.5\%) experienced HBV reactivation and HCC recurred in three patients $(7.7 \%)$. Three patients underwent re-transplantation because of graft failure that was not related to HBV.

Conclusions: In the era of NA for HBV, HBsAg (+) liver graft can be used safely with expanding donor pool in HBV endemic areas.

Corresponding author: YoungRok Choi

E-mail: choiyoungrok@gmail.com 